\title{
GEOCONTEXT AND CHCONTEXT GEOGRAPHIC INFORMATION IN CULTURAL HERITAGE
}

\author{
L. Marsicano ${ }^{\text {a }}$, M. Montanari ${ }^{b}$ \\ ${ }^{a}$ Open History Map, Bologna, Italy - lucia.marsicano@openhistorymap.org \\ b Open History Map, Bologna, Italy - marco.montanari@openhistorymap.org
}

\section{Commission IV, WG IV/4}

KEY WORDS: cultural heritage, web platforms, code generation, interpreters, geographic visualization, geographic content

\begin{abstract}
:
Internet presence and applications are central for modern museums and cultural institutions. Even more it is important to facilitate and standardize the user experience in order to create a standard quality level and, for the institutions, it is important to simplify configuration operations bound to the creation of the visualized elements and the explorations of the spaces. This demo covers both the technologies underneath the GeoContext tool and the CHContext visualization generator as well as the possible targets and applications.

The final elements will cover possible enhancements already in the works.
\end{abstract}

\section{INTRODUCTION}

\subsection{What are GeoContext and CHContext}

GeoContext is a geographic dataset contextualizer and visualizer. In a world where data is constantly produced it becomes every day more difficult to understand and contextualize such data. For this reason it is paramount to locate and confront new data with pre-existing already analyzed and metered information. In wake of these considerations we created GeoContext, as a manner to standardize data contextualization and referencing.

When trying to expose and valorize cultural heritage it is paramount to contextualize both in time and space the elements to be exposed. For this reason, based on the GeoContext platform, we created the CHContext toolset, enabling administrators to define a storytelling within the points and geographic elements displayed within the geocontext toolset. In addition to that, CHContext standardizes the itneractions between online tools and between data structures and datasets.

\section{USE CASES}

\footnotetext{
${ }^{1} \mathrm{http}: / /$ www.appasseggio.it/index.php?it/161/appassegg io-con-gli-etruschi
}

\subsection{Usage areas}

As previously stated, GeoContext aims to be an interface for geographical information giving the possibility to contextualize any kind of data.

The last few years have seen an incredible rise in the awareness of the connection between cultural heritage and its geographic context, and this awareness is also present in the institutions that manage and protect our culture and history. This is even truer in Italy, where in the last years there has been a rediscovery of the concept of Ecomuseums. This rediscovery as spaces, not only physical, of shared and coordinated management of the cultural heritage bringing the geographical, historical and environmental context - the one that made a specific area an element of cultural heritage - to the attention of both researchers as well as passionate visitors alike (Murtas, 2017).

Further, it is even more common for cultural institutions to publish mobile applications that enable the fruition in a larger territorial scale. Sometimes, these applications are created in coordination with Overseers, like in the case of APPasseggio ${ }^{1}$, which collects several Points of interest bound to local Etruscan history.

These apps are usually highly customized and vertical on a specific problem, becoming deeply crafty and artisanal, 
thus losing the possibility to be affordable for small institutions.

\subsection{First prototypes}

The CHContext experience starts from an experiment in the creation of a geographic information collection platform enabling users to explore and "live" the cultural heritage surrounding them. The first step was made for the creation of the treasure hunt at the Bank of Naples Foundation, which happened in june 2017. The treasure hunt had the starting point at the Historical Archive of the Bank of Naples Foundation and, to join the hunt, it was necessary for the players to install a custom application. GPS and QRCode readers were required to find the hints and solve the steps. The event, with a highly cultural structure, required the creation of a custom dedicated product because there was no platform or tool already available and useful for such an activity.

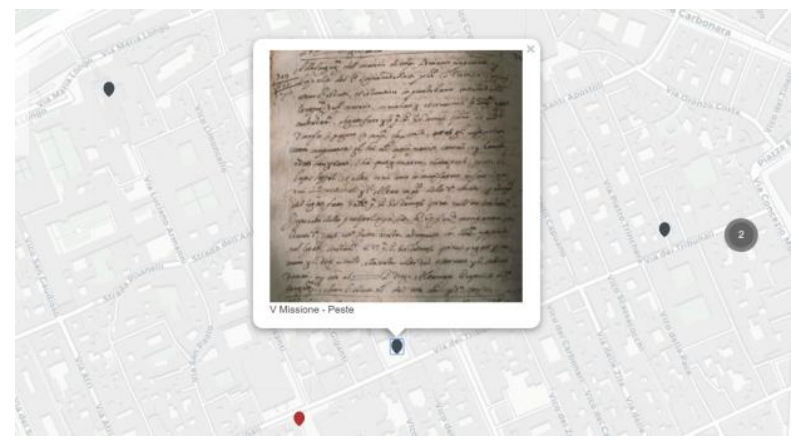

Figure 1. Cultural Treasure Hunt

The creation of such prototype was central in the development of both the GeoContext as well as the CHContext platforms. The treasure hunt is just one of the several cultural gamified activities that require an exploration of a more or less large area where points of interest can be explored either actively or passively. Even though such an approach is lately becoming more and more common there is not yet a specific solution or a shared platform to operate in this direction.

\section{ARCHITECTURE}

\subsection{The CHContext Platform}

The previously described experiences have been crucial in the definition of the features of the CHContext Platform. The typical elements in applications such as the ones before mentioned are the ones defined in Figure 2.

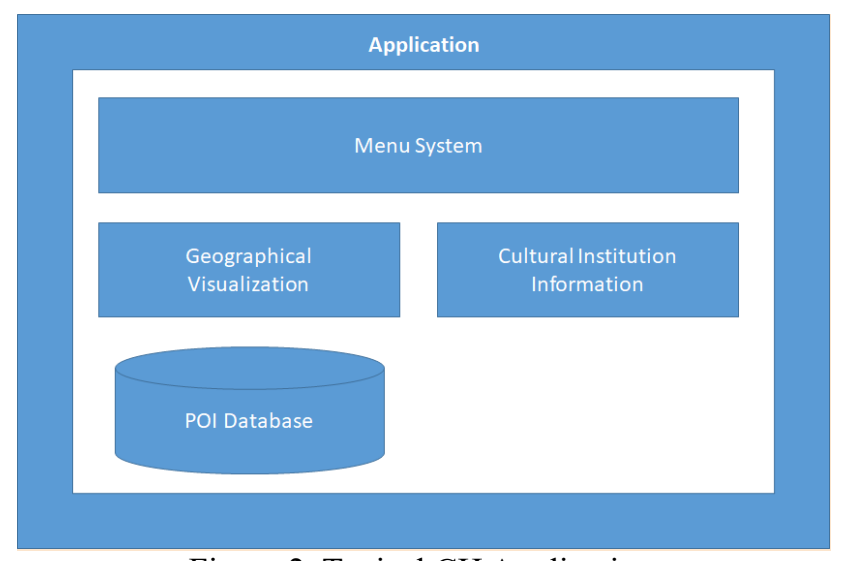

Figure 2. Typical CH Application

Applying a standard "divide and conquer" approach, the main elements can be extracted and what remains is an abstract application descriptor, a set of points of interest and a very small set of custom items that can be easily managed through a common CMS architecture.

\subsection{CHContext Architecture}

The architecture of the platform does not rely on a classic 3-tier system but rather an enhanced meta-service based model (Montanari, 2018) where various separate components use the classic http protocol as a communication middleware coordinated by one simple web orchestration API that exposes the specific domain items through a custom front end. The whole infrastructure is managed as separate vertical components exposing simple RESTful APIs, all domain specific.

All the basic components are managed via a docker swarm infrastructure relying on independent docker stacks. All of the stacks are metered and can be monitored in order to give usage feedback on specific elements of the stacks.

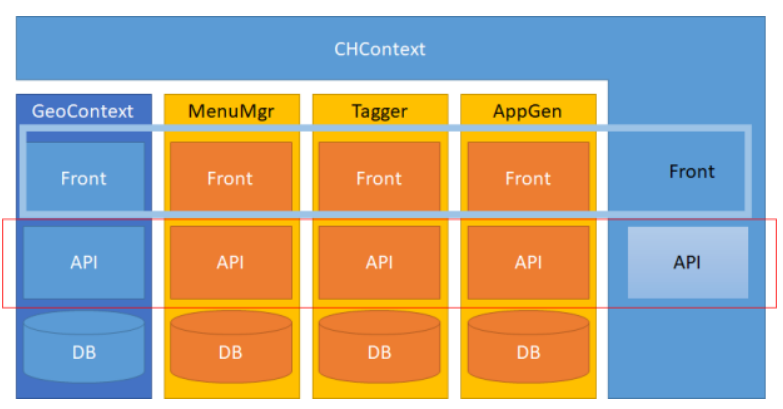

Figure 3. CHContext Architecture

The CHContext platform gives the general structure of the components to be used during the application generation. For example, it defines the properties to be used in GeoContext to define which elements shall be hidden in a treasurehunt, and which not. The Menu Manager enables the creation of a full-fledget platform independent menu structure, for users to create complex application interactions. The defined structure can be used to simply 
move around a menu tree or to store a set of settings to be used to initialize specific activities.

The Tagger tool is a language-agnostic object tagging element, enabling the creation of language independent QR-Code and BLE driven descriptive material for objects and items in museums and areas.

All these components have UI elements and standardized interactions to be integrated into Angular5 or Android or iOS apps simplifying the creation of tools to the definition of configuration files and the usage of specific application schematics to simplify the buildup of codable applications.

\subsection{CHContext Roles}

Administrators and end users have the same requirements on a saas platform, considering their main difference is the possibility to alter or use the resources the saas platform offers. In the GeoContext and CHContext case, the resources managed are

- Via GeoContext, the physical visualization of the elements covered by the dataset;

- Via MenuMaker, the structure of the menus;

- Via Tagger, the content of the descriptions of the exposed items;

- Via AppGen, the general structure of the application to be generated.

The administrative role is managed via groups, meaning a team can manage any part of the configuration, without the risk of blocking the configuration or the macromanagement of the apps.

\section{DEMO}

\subsection{Archaeotrekking}

With the architecture in mind, the following step was to create an app supporting an archaeotrekking event during the 2017 earth week. The track had the topic of the pleistocenic sites of the northeastern area of Rome, covering both still existing as well as lost archaeological areas. The material for the creation of the app was preexisting GIS data, with a simple database given by the Museo di Casal de'pazzi that organized the event.

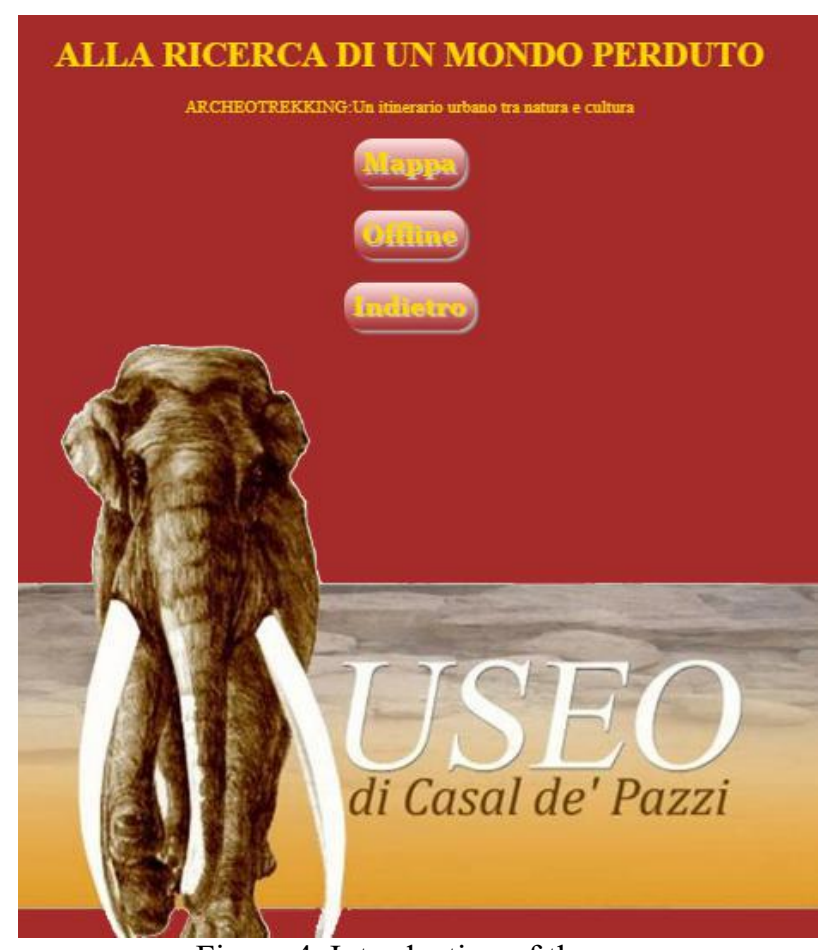

Figure 4. Introduction of the app

The app gives the user, on a geographic background, information on her relative position in a large environmentally important area with specific information packages on the various known pleistocenic sites. Three of them are part of an institutionalized visit and visitors can decide to follow the predefined trip or browse in the area exploring the interest points directly. The information about the POIs is in many forms: textual, multimedia and in interactive $3 \mathrm{D}$ models.

\subsection{Ghosts in Rome}

CHContext has been used in a project to uncover the mystic tradition of the city of Rome. Following the tradition, Rome is "infested" by ghosts of various characters from various social levels and historical moments. The center, from Castel Sant'Angelo to Piazza di Spagna is target of these phenomena that somehow interleave with the long story of Rome and are embedded in its legends.

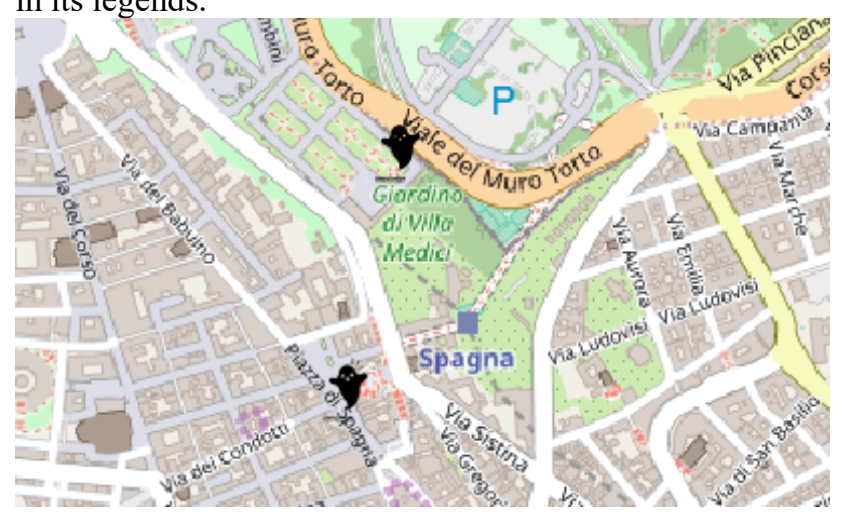

Figure 5. Two sample locations of "Ghosts in Rome" 
The app created is both for end-users to explore autonomously, but has a "layer" for guides, enabling them to give additional information to users.

The mapped places are 15, enabling users to explore the Area and get a glimpse into the various cities that Rome has been during the millennia.

\section{FUTURE WORK}

\subsection{Work in progress}

The GeoContext platform enables the integration into CHContext of several different datasources. Integration with crowdsourcing platforms aiming at cultural heritage and cultural representation are undergoing discussing with with projects like Mappi-Na, enabling the definition of tracks to discover the Street Art in Bologna.

From a technical point of view, the app generation description and the process are being analyzed, enabling the further customization of the app pages and the app srtucture.

In addition to this, the work is now looking into the integration of possible additional vertical services, enabling social integration, sharing of created tracks, crowdsourcing info on the points of interest looking at projects like Adopt a Hydrant ${ }^{2}$ and enabling the easy creation of complex geographically aware applications through the standardization of the interacitons.

\section{BIBLIOGRAPHY}

Montanari, M., 2018. Medium. [Online]

Available at: https://medium.com/p/c3e2f35605f9

[Accessed 2904 2018].

Murtas, D., 2017. Mappe di Comunità. [Online]

Available at: https://visionscarto.net/mappe-di-comunita

\footnotetext{
${ }^{2}$ https://www.codeforamerica.org/past-projects/adopt-ahydrant
} 